\title{
Assessment and management of itchy skin in pregnancy
}

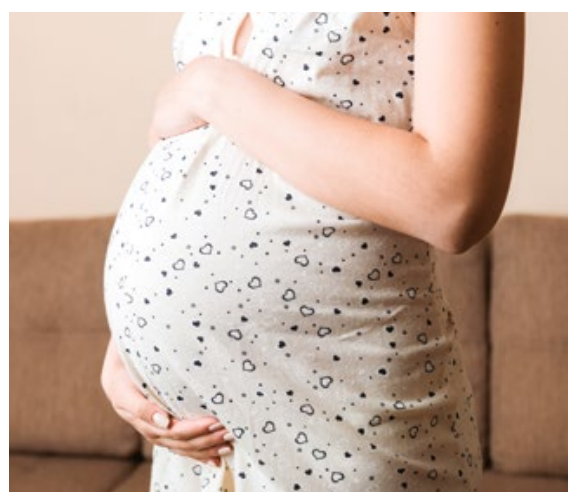

CPD

\section{Sarajane Ting, Rosemary Nixon}

\section{Background}

Women with rashes or itchy skin during pregnancy will often present initially to the general practitioner. Knowledge of the specific dermatoses of pregnancy will assist in diagnosis, management and, importantly, facilitation of timely escalation of care of conditions that can potentially affect the fetus.

\section{Objective}

The aim of this article is to provide a diagnostic framework for approaching a pruritic rash during pregnancy as well as a helpful summary of management of pregnancy-specific dermatoses. It will assist clinicians in the identification of specific dermatoses that pose fetal risks.

\section{Discussion}

In addition to considering non-pregnancy specific conditions when approaching pruritus or a pruritic rash in pregnancy, it is important that clinicians also consider pregnancy-specific dermatoses, which have been reclassified into four categories: polymorphic eruption of pregnancy, pemphigoid gestationis, intrahepatic cholestasis of pregnancy (ICP) and atopic eruption of pregnancy. Unlike the other dermatoses, ICP begins with pruritus, and skin changes are secondary. ICP and pemphigoid gestationis are associated with fetal risks such as prematurity and stillbirth.
PREGNANT WOMEN with itchy skin or a rash will often seek help from their general practitioners (GPs) initially. This article suggests a framework for the assessment of these presentations (Figure 1). While pre-existing skin conditions and nonpregnancy specific conditions such as psoriasis, urticaria, drug eruptions, contact dermatitis and scabies are important to consider, there is a specific group of conditions (called the specific dermatoses of pregnancy) that the clinician should consider when assessing a pregnant woman presenting with pruritus alone or a pruritic rash. The specific dermatoses of pregnancy are conditions that are pruritic and, by definition, only occur during pregnancy. These conditions have undergone reclassification into four main categories, namely polymorphic eruption of pregnancy (PEP), pemphigoid gestationis, intrahepatic cholestasis of pregnancy (ICP) and atopic eruption of pregnancy (AEP). ${ }^{1}$ Given clinicians are generally familiar with pre-existing conditions or cutaneous disorders affected by pregnancy while the specific dermatoses of pregnancy may be relatively more poorly understood, the specific dermatoses of pregnancy conditions are discussed in more detail in this article. Furthermore, potential fetal risks are emphasised to raise awareness among clinicians, which will assist with facilitation of timely management (Table 1). While ICP presents initially with intense itch that may be followed by skin manifestations due to scratching, ICP is included in discussion in this article as it is an important condition to consider when assessing any pregnant woman presenting with pruritus, given its associated potential fetal risks.

\section{Clinical assessment}

Potential differential diagnoses to consider in a pregnant woman with pruritus and rash are included in Figure 1. The main dermatoses of pregnancy to exclude are pemphigoid gestationis and ICP given their associated fetal risks. Features that would support a diagnosis of pemphigoid gestationis include an intensely itchy vesicular or bullous rash on the abdomen area with umbilical involvement, history of pemphigoid gestationis in previous pregnancies and presence of other autoimmune conditions. If pemphigoid gestationis is suspected, a biopsy should be performed to confirm the diagnosis. ICP is unique in that intense itch precedes any rash. Physical examination will reveal only secondary lesions due to scratching. If ICP is suspected, the diagnosis can be confirmed with elevated serum bile 
acid levels. Pemphigoid gestationis and ICP require timely specialist obstetric involvement, ideally within one week. Multidisciplinary team involvement is encouraged in approaching diagnosis and management of these conditions, involving the GP, dermatologist and obstetric team. The dermatoses of pregnancy are elaborated further in detail below.

\section{Polymorphic eruption of pregnancy}

PEP, or pruritic urticarial papules and plaques of pregnancy (PUPP), occurs most commonly in primigravid women in the third trimester of pregnancy but uncommonly can present after delivery. ${ }^{1,2}$ It is relatively common and affects approximately one in 200 pregnancies. $^{3}$ It has been suggested that the pathological basis of PEP is related to an inflammatory process triggered by rapid abdominal distension, which could explain the association of PEP with excessive maternal weight gain and multiple pregnancies. ${ }^{2,4}$

PEP presents with intensely pruritic urticarial papules before coalescing to form plaques (Figure 2). It initially starts on the abdominal wall within striae and spreads to the extremities, but the face, palms and soles are rarely involved. The periumbilical area is classically spared. ${ }^{2}$ PEP is a clinical diagnosis, and skin biopsy is rarely required. Some patients can develop vesicles and targetoid lesions, which can be difficult to distinguish from pemphigoid gestationis. In such cases, a biopsy should be performed for histology and direct immunofluorescence studies. As opposed to pemphigoid gestationis, direct immunofluorescence studies are negative in PEP. ${ }^{5}$ Direct immunofluorescence is used for demonstration of tissue-bound antibody and complement, and it can be requested with histology after taking a biopsy of the edge of the vesicle. The specimen for direct immunofluorescence should be placed in saline or a special immunofluorescence medium (not formalin).

PEP is self-limiting and tends to resolve spontaneously 4-6 weeks after its onset, or days to weeks after delivery. ${ }^{2,6}$ It does not have any adverse effects on the fetus and does not tend to recur in subsequent pregnancies. ${ }^{4,7}$ Treatment is aimed at symptom relief with topical corticosteroids and antihistamines. In more severe cases, systemic corticosteroids can be used. Prednisolone at a dose of 40-60 mg daily (tapering over a few days) is the systemic steroid of choice and is generally considered safe in pregnancy, especially as only short-term therapy is usually required to achieve control. ${ }^{5}$ There have been rare reports of severe intractable PEP prompting early delivery $;^{8,9}$ however, this is rarely required, and controversy remains regarding the true effect of delivery on resolution of symptoms. ${ }^{2,10}$

\section{Pemphigoid gestationis}

It is important to distinguish the self-limiting PEP from pemphigoid gestationis, previously known as herpes gestationis, because of the potential fetal risks associated with pemphigoid gestationis. Pemphigoid gestationis is an autoimmune blistering disease of pregnancy that has been associated with other autoimmune conditions such as

Table 1. Summary of pregnancy-specific dermatoses

\begin{tabular}{|c|c|c|c|c|}
\hline $\begin{array}{l}\text { Pregnancy-specific } \\
\text { dermatosis }\end{array}$ & Clinical presentation & Laboratory investigations & Treatment & Fetal risk \\
\hline $\begin{array}{l}\text { Polymorphic eruption } \\
\text { of pregnancy }\end{array}$ & $\begin{array}{l}\text { Urticarial papules and plaques initially } \\
\text { within abdominal striae and spreading } \\
\text { to extremities; periumbilical sparing }\end{array}$ & None & $\begin{array}{l}\text { Topical steroids, } \\
\text { antihistamines, rarely } \\
\text { systemic steroids }\end{array}$ & None \\
\hline $\begin{array}{l}\text { Pemphigoid } \\
\text { gestationis }\end{array}$ & $\begin{array}{l}\text { Vesiculobullous eruption starting } \\
\text { on abdomen and spreading to } \\
\text { extremities; periumbilical involvement }\end{array}$ & $\begin{array}{l}\text { Histopathology: } \\
\text { subepidermal blister } \\
\text { Direct immunofluorescence: } \\
\text { linear C3 deposition along } \\
\text { basement membrane }\end{array}$ & Systemic steroids & $\begin{array}{l}\text { Prematurity, } \\
\text { SGA, stillbirth }\end{array}$ \\
\hline $\begin{array}{l}\text { Intrahepatic } \\
\text { cholestasis of } \\
\text { pregnancy }\end{array}$ & $\begin{array}{l}\text { No primary lesions; generalised } \\
\text { pruritus involving palms and soles }\end{array}$ & $\begin{array}{l}\text { Elevated bile acid levels, mildly } \\
\text { deranged LFTs }\end{array}$ & Ursodeoxycholic acid & $\begin{array}{l}\text { Prematurity, } \\
\text { fetal distress, } \\
\text { stillbirth, FDIU }\end{array}$ \\
\hline $\begin{array}{l}\text { Atopic eruption } \\
\text { of pregnancy }\end{array}$ & $\begin{array}{l}\text { Eczema in pregnancy: eczematous } \\
\text { lesions on flexural surfaces, } \\
\text { extremities, trunk } \\
\text { Prurigo of pregnancy: excoriated } \\
\text { papules and nodules on extensor } \\
\text { surfaces of limbs and trunk } \\
\text { Pruritic folliculitis of pregnancy: } \\
\text { papules on trunk before forming } \\
\text { pustules }\end{array}$ & Elevated IgE levels & $\begin{array}{l}\text { Emollients, topical } \\
\text { steroids, antihistamines, } \\
\text { UVB therapy }\end{array}$ & None \\
\hline
\end{tabular}

C3, complement 3; FDIU, fetal death in utero; IgE, immunoglobulin E; LFTs, liver function tests; SGA, small-for-gestational-age; UVB, ultraviolet B 


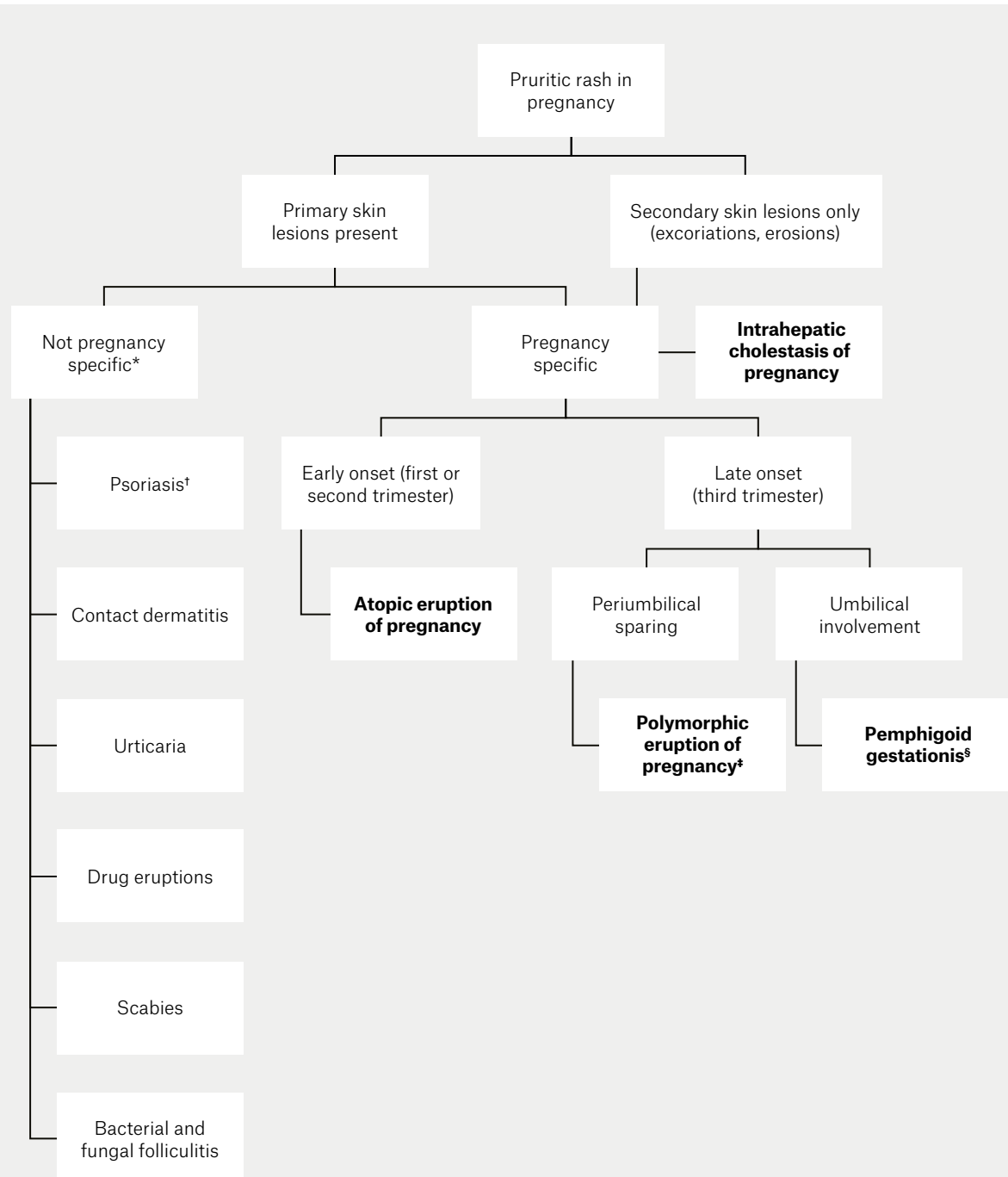

Figure 1. A diagnostic framework to approaching rash and pruritus in pregnancy

*Included are suggested differential diagnoses to consider; however, this is not an exhaustive list.

tIt is important to specifically consider pustular psoriasis of pregnancy given its maternal and fetal risks.

FPolymorphic eruption of pregnancy tends to occur mostly in primigravid women. In multigravid women, consider other causes.

sPemphigoid gestationis usually presents in the third trimester but can occasionally present earlier.

Grave's disease, vitiligo and pernicious anaemia. ${ }^{11}$ It most commonly begins in late pregnancy but, unlike PEP, may occur earlier. It resolves within a few weeks after delivery. ${ }^{12}$ It tends to recur in subsequent pregnancies and can relapse with menstruation and oral contraceptive use. ${ }^{4,13}$ Although well described, pemphigoid gestationis is rare, affecting approximately one in 50,000 pregnancies. ${ }^{14}$

Pemphigoid gestationis presents with papules, plaques, vesicles and bullae that first appear on the abdominal wall and spread to the extremities. The lesions are intensely pruritic. Unlike PEP, the umbilical area is involved (Figure 3). The face and mucous membranes are rarely involved..$^{12,15}$ The diagnosis of pemphigoid gestationis is confirmed by histopathology and, most importantly, direct immunofluorescence studies, which show linear deposits of complement $3+$ +-immunoglobulin (Ig) G along the basement membrane. ${ }^{11,16}$
Pemphigoid gestationis is associated with prematurity, small-for-gestational-age infants and stillbirth. ${ }^{17}$ Close antenatal surveillance and fetal monitoring are recommended for pregnant women with pemphigoid gestationis. Treatment is aimed at managing pruritus and preventing further formation of blisters. ${ }^{5}$ In mild cases, topical corticosteroids can be used; however, systemic steroids, such as prednisolone $0.5-1 \mathrm{mg} / \mathrm{kg} /$ day, are usually considered first-line therapy. ${ }^{13}$ 
Multidisciplinary care, including dermatology input, should be considered for patients with suspected pemphigoid gestationis, especially in patients with refractory pemphigoid gestationis.

\section{Intrahepatic cholestasis of pregnancy}

ICP is the only dermatosis of pregnancy that presents initially with pruritus without any primary skin lesions. Skin manifestations, such as excoriations, erosions and prurigo nodules, result from scratching due to severe pruritus. It is crucial to recognise ICP as it can be associated with poor fetal outcomes. Although the prevalence of ICP varies worldwide (between $0.3 \%$ and $5.6 \%$ of pregnancies) depending on geographical and ethnic factors, an Australian study has shown a prevalence of $0.7 \% .^{18}$

ICP usually presents in the second or third trimester with sudden-onset pruritus all over the body, including the palms and soles. The pruritus tends to resolve postpartum; however, it can recur in subsequent pregnancies. ${ }^{19,20}$ The diagnosis is based on the combination of severe pruritus and laboratory investigations, which will show elevated bile acid levels. ${ }^{21}$ While alkaline phosphatase levels are normally elevated in pregnant women, liver function tests may also show mildly abnormal aspartate and alanine transaminase levels. ${ }^{19}$ The mainstay of

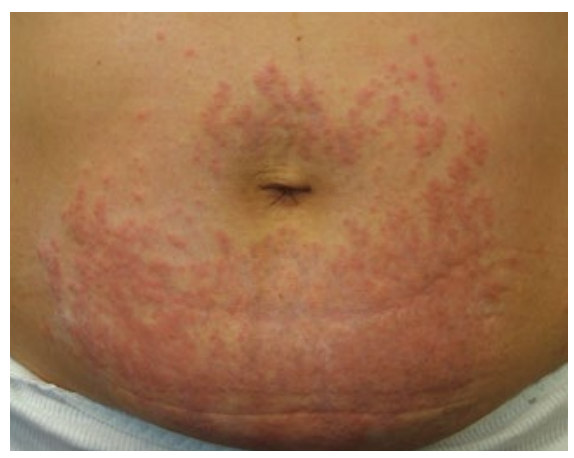

Figure 2. Polymorphic eruption of pregnancy: pruritic papules on the abdominal area coalescing to form plaques, with periumbilical sparing treatment for ICP is ursodeoxycholic acid, a naturally occurring bile acid given at a dose of $15 \mathrm{mg} / \mathrm{kg} /$ day or $1 \mathrm{~g} /$ day orally to manage pruritus, reduce bile acid levels and improve fetal outcomes. ${ }^{22,23}$

ICP is associated with pregnancy risks such as prematurity, fetal distress and stillbirth. ${ }^{24}$ Fetal death predominantly occurs around 38 weeks of gestation; therefore, it is usually recommended that delivery occurs by 38 weeks. ${ }^{25}$ In severe cases, earlier delivery may be indicated. ${ }^{26}$ ICP is often closely managed with maternal fetal medicine specialists.

\section{Atopic eruption of pregnancy}

AEP is a term coined to define a group of conditions that includes eczema in pregnancy, prurigo of pregnancy and pruritic folliculitis of pregnancy (PFP). ${ }^{1}$ However, the recent inclusion of prurigo of pregnancy and PFP under AEP is controversial as they have not been conclusively shown to have an atopic diathesis. ${ }^{26}$ They share similarities of presenting with itchy eczematous lesions with a benign prognosis with no maternal or fetal risks. ${ }^{1,5}$ In contrast to the other dermatoses of pregnancy, AEP tends to present earlier in pregnancy, in the first or second trimester.

Eczema in pregnancy is the most common dermatosis of pregnancy, affecting almost $50 \%$ of patients who present with dermatoses of pregnancy. A woman

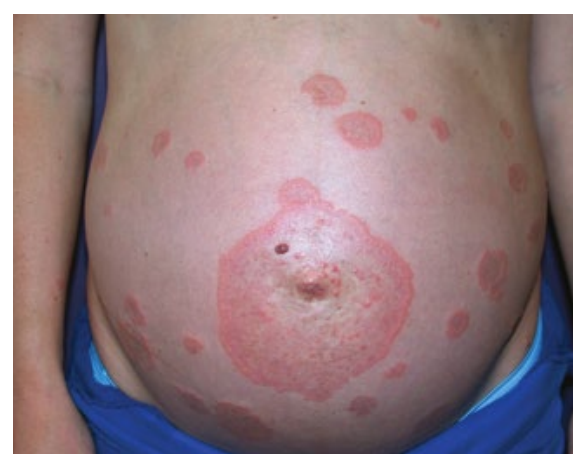

Figure 3. Pemphigoid gestationis: plaques and vesicles that begin on the abdominal wall before spreading, with periumbilical involvement with eczema in pregnancy may present with either eczematous skin changes for the first time if they are a patient with an atopic background or, less commonly, with exacerbation of pre-existing atopic dermatitis. Sites involved include classic flexural surfaces as well as the extremities and trunk. ${ }^{1}$ Prurigo of pregnancy affects approximately one in 300 pregnancies. ${ }^{4}$ The characteristic lesions of prurigo of pregnancy are erythematous excoriated papules or nodules on the trunk and extensor surfaces of the arms and legs (Figure 4). The lesions can sometimes resemble prurigo nodules. ${ }^{1,27}$ ICP should be considered as a differential diagnosis; however, in contrast to prurigo of pregnancy, pruritus precedes any rash in ICP. PFP is rare and affects one in 3000 pregnancies. ${ }^{28}$ It presents initially with papules on the trunk that evolve to form generalised pustules and follicular papules. ${ }^{26}$ Culture studies should be performed to exclude bacterial or fungal folliculitis.

The diagnosis of AEP is largely clinical, as there are no diagnostic tests. Histopathology is non-specific, and direct immunofluorescence studies are negative; however, serum IgE levels may be raised. ${ }^{1}$ AEP tends to resolve early in the postpartum period. Treatment of AEP is symptomatic with the use of emollients, topical corticosteroids and antihistamines. In severe refractory cases, a short course of systemic corticosteroids or narrow-band ultraviolet B therapy can be used. ${ }^{29}$

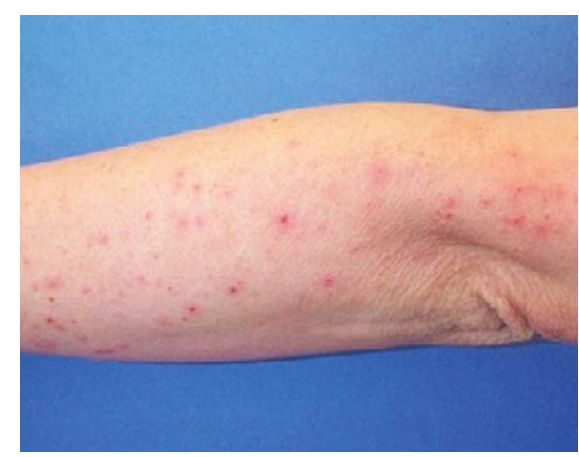

Figure 4. Prurigo of pregnancy: excoriated papules on the extensor surface of the arm 


\section{Pustular psoriasis of pregnancy}

Pustular psoriasis of pregnancy (PPP), or impetigo herpetiformis, is considered a variant of generalised pustular psoriasis rather than a specific dermatosis of pregnancy. ${ }^{30,31}$ However, PPP merits special mention to encourage clinicians to consider this condition given its maternal and fetal risks. PPP is rare, and its prevalence is unknown because of the paucity of reported cases.

PPP typically presents in the third trimester of pregnancy and can recur in subsequent pregnancies. ${ }^{32}$ It is characterised by multiple sterile pustules in a circinate pattern, usually starting in the intertriginous areas and spreading centrifugally. As opposed to the other dermatoses of pregnancy, it is associated with systemic symptoms such as fever, chills, vomiting and diarrhea. ${ }^{33}$ Laboratory tests show neutrophilia, elevated inflammatory markers, hypocalcaemia, hypoparathyroidism, low phosphate and low vitamin D levels. Blood cultures and cultures of pustules are negative. ${ }^{34}$ Histopathology demonstrates spongiform pustules with neutrophils, psoriasiform hyperplasia and parakeratosis. Direct immunofluorescence is negative. ${ }^{33}$ First-line treatment options include systemic corticosteroids, cyclosporine, infliximab, topical corticosteroids and topical calcipotriol. ${ }^{35}$ With early recognition and treatment, maternal risks such as delirium and tetany secondary to hypocalcemia can be avoided. Fetal prognosis is less favourable, with poor outcomes reported including intrauterine growth restriction and stillbirth. ${ }^{36}$

\section{Conclusion}

In summary, an awareness of pregnancyspecific dermatoses will assist the GP in the clinical approach to a pregnant woman presenting with pruritus and rash. Importantly, pemphigoid gestationis and ICP are diagnoses that should not be missed because of the potential adverse fetal outcomes. It is common for pemphigoid gestationis and ICP to be managed in conjunction with specialist dermatology and obstetric or maternal fetal medicine teams to optimise fetal outcomes. Although not specifically a dermatosis of pregnancy, PPP should also be considered given its potential maternal and fetal risks.

\section{Authors}

Sarajane Ting MBBS (Hons), BMedSc, FRACGP, $\mathrm{DCH}$, General Practitioner, Tas

Rosemary Nixon AM BSc (Hons), MBBS, MPH, FACD, FAFOEM, Consultant Dermatologist and Occupational Physician; Adjunct Clinical Associate Professor, Monash University, Vic; Honorary Associate Professor, University of Melbourne, Vic; Director, Occupational Dermatology Research and Education Centre, Carlton, Vic

Competing interests: None.

Funding: None.

Provenance and peer review: Not commissioned, externally peer reviewed.

Correspondence to:

sjaneting@gmail.com

\section{Acknowledgements}

The authors would like to acknowledge Associate Professor Amanda Oakley for providing the images in this article.

\section{References}

1. Ambros-Rudolph $\mathrm{CM}$, Müllegger RR, Vaughan-Jones SA, Kerl H, Black MM. The specific dermatoses of pregnancy revisited and reclassified: Results of a retrospective two-center study on 505 pregnant patients. J Am Acad Dermatol 2006;54(3):395-404. doi: 10.1016/j. jaad.2005.12.012

2. Rudolph CM, Al-Fares S, Vaughan-Jones SA, Müllegger RR, Kerl H, Black MM. Polymorphic eruption of pregnancy: Clinicopathology and potential trigger factors in 181 patients. $\mathrm{Br} J$ Dermatol 2006;154(1):54-60. doi: 10.1111/j.13652133.2005.06856.x

3. Vaughan Jones S, Ambros-Rudolph C, Nelson-Piercy C. Skin disease in pregnancy. BMJ 2014;348:g3489. doi: 10.1136/bmj.g3489.

4. Kroumpouzos G, Cohen LM. Specific dermatoses of pregnancy: An evidence-based systematic review. Am J Obstet Gynecol 2003;188(4):1083-92. doi: 10.1067/mob.2003.129.

5. Ambros-Rudolph CM. Dermatoses of pregnancy Clues to diagnosis, fetal risk and therapy. Ann Dermatol 2011;23(3):265-75. doi: 10.5021/ ad.2011.23.3.265.

6. Brandão $P$, Sousa-Faria $B$, Marinho $C$, Vieira-Enes P, Melo A, Mota L. Polymorphic eruption of pregnancy: Review of literature. J Obstet Gynaecol 2017;37(2):137-40. doi: 10.1080/01443615.2016.1225019.

7. Ohel I, Levy A, Silberstein T, Holcberg G, Sheiner E. Pregnancy outcome of patients with pruritic urticarial papules and plaques of pregnancy. J Matern Fetal Neonatal Med 2006;19(5):305-08. doi: 10.1080/14767050600590573.

8. Beltrani VP, Beltrani VS. Pruritic urticarial papules and plaques of pregnancy: A severe case requiring early delivery for relief of symptoms. J Am Acad Dermatol 1992;26(2 Pt 1):266-67. doi: 10.1016/ s0190-9622(08)80308-8.

9. Bunker CB, Erskine K, Rustin MH, Gilkes JJ. Severe polymorphic eruption of pregnancy occurring in twin pregnancies. Clin Exp Dermatol 1990;15(3):228-31. doi: 10.1111/j.1365-2230.1990. tb02078.x.

10. Carruthers A. Pruritic urticarial papules and plaques of pregnancy. J Am Acad Dermatol 1993;29(1):125. doi: 10.1016/s01909622(08)81820-8.

11. Shimanovich I, Bröcker EB, Zillikens D. Pemphigoid gestationis: New insights into the pathogenesis lead to novel diagnostic tools. BJOG 2002;109(9):970-76. doi: 10.1111/j.14710528.2002.01016.x.

12. Semkova K, Black M. Pemphigoid gestationis: Current insights into pathogenesis and treatment. Eur J Obstet Gynecol Reprod Biol 2009;145(2):138-44. doi: 10.1016/j. ejogrb.2009.05.012.

13. Patsatsi A, Vavilis D, Tsikeloudi M, Kalabalikis D, Sotiriadis D. Refractory pemphigoid gestationis postpartum. Acta Obstet Gynecol Scand 2012;91(5):636-37. doi: 10.1111/j.16000412.2012.01379.x.

14. Yancey KB. Herpes gestationis. Dermatol Clin 1990;8(4):727-35.

15. Jenkins RE, Hern S, Black MM. Clinical features and management of 87 patients with pemphigoid gestationis. Clin Exp Dermatol 1999;24(4):255-59. doi: 10.1046/j.1365-2230.1999.00472.x.

16. Intong LR, Murrell DF. Pemphigoid gestationis: Pathogenesis and clinical features. Dermatol Clin 2011;29(3):447-52, ix. doi: 10.1016/j. det.2011.03.002.

17. Chi CC, Wang SH, Charles-Holmes R, et al. Pemphigoid gestationis: Early onset and blister formation are associated with adverse pregnancy outcomes. Br J Dermatol 2009;160(6):1222-28. doi: 10.1111/j.1365-2133.2009.09086.x.

18. Gardiner FW, McCuaig R, Arthur C, et al. The prevalence and pregnancy outcomes of intrahepatic cholestasis of pregnancy: A retrospective clinical audit review. Obstet Med 2019;12(3):123-28. doi: 10.1177/1753495X18797749.

19. Riely CA, Bacq Y. Intrahepatic cholestasis of pregnancy. Clin Liver Dis 2004;8(1):167-76. doi: 10.1016/S1089-3261(03)00131-4.

20. Pathak B, Sheibani L, Lee RH. Cholestasis of pregnancy. Obstet Gynecol Clin North Am 2010;37(2):269-82. doi: 10.1016/j.ogc.2010.02.011.

21. Glantz A, Marschall HU, Mattsson LA. Intrahepatic cholestasis of pregnancy: Relationships between bile acid levels and fetal complication rates. Hepatology 2004;40(2):467-74. doi: 10.1002/ hep.20336.

22. Kondrackiene J, Beuers U, Kupcinskas L. Efficacy and safety of ursodeoxycholic acid versus cholestyramine in intrahepatic cholestasis of pregnancy. Gastroenterology 2005;129(3):894-901. doi: 10.1053/j.gastro.2005.06.019.

23. Chappell LC, Gurung V, Seed PT, et al. Ursodeoxycholic acid versus placebo, and early term delivery versus expectant management, in women with intrahepatic cholestasis of pregnancy: Semifactorial randomised clinical trial. BMJ 2012;344:e3799. doi: 10.1136/bmj.e3799.

24. Lammert F, Marschall HU, Glantz A, Matern S. Intrahepatic cholestasis of pregnancy: Molecular pathogenesis, diagnosis and management. J Hepatol 2000;33(6):1012-21. doi: 10.1016/s01688278(00)80139-7.

25. Williamson C, Geenes V. Intrahepatic cholestasis of pregnancy. Obstet Gynecol 2014;124(1):120-33. doi: 10.1097/AOG.0000000000000346.

26. Roth MM. Pregnancy dermatoses: Diagnosis, management, and controversies. Am J Clin 
Dermatol 2011;12(1):25-41. doi: 10.2165/11532010000000000-00000.

27. Kroumpouzos G, Cohen LM. Dermatoses of pregnancy. J Am Acad Dermatol 2001;45(1):1-19. doi: $10.1067 / \mathrm{mjd} .2001 .114595$.

28. Roger D, Vaillant L, Fignon A, et al. Specific pruritic diseases of pregnancy. A prospective study of 3192 pregnant women. Arch Dermatol 1994;130(6):734-39.

29. Reed J, George S. Pruritic folliculitis of pregnancy treated with narrowband (TL-01) ultraviolet B phototherapy. Br J Dermatol 1999;141(1):177-79. doi: 10.1046/j.1365-2133.1999.02950.x.

30. Trivedi MK, Vaughn AR, Murase JE. Pustular psoriasis of pregnancy: Current perspectives. Int J Womens Health 2018;10:109-15. doi: 10.2147/ IJWH.S125784.

31. Chang SE, Kim HH, Choi JH, Sung KJ, Moon KC, Koh JK. Impetigo herpetiformis followed by generalized pustular psoriasis: More evidence of same disease entity. Int J Dermatol 2003;42(9):754-55. doi: 10.1046/j.13654362.2003.01998.x

32. Shah A, Makhecha M. Pustular psoriasis of pregnancy with acrodermatitis continua of hallopeau. Indian J Dermatol 2016;61(1):123. doi: 10.4103/0019-5154.174149.

33. Breier-Maly J, Ortel B, Breier F, Schmidt JB, Hönigsmann $\mathrm{H}$. Generalized pustular psoriasis of pregnancy (impetigo herpetiformis). Dermatology 1999;198(1):61-64. doi: 10.1159/000018066.

34. Flynn A, Burke N, Byrne B, Gleeson N, Wynne B, Barnes $L$. Two case reports of generalized pustular psoriasis of pregnancy: Different outcomes. Obstet Med 2016;9(2):55-59. doi: 10.1177/1753495X15626623.

35. Robinson A, Van Voorhees AS, Hsu S, et al. Treatment of pustular psoriasis: From the Medical Board of the National Psoriasis Foundation. J Am Acad Dermatol 2012;67(2):279-88. doi: 10.1016/j. jaad.2011.01.032

36. Lim KS, Tang MB, Ng PP. Impetigo herpetiformis $A$ rare dermatosis of pregnancy associated with prenatal complications. Ann Acad Med Singap 2005;34(9):565-68. 